Accepted for publication in Neural Computation

\title{
How to pretend that correlated variables are independent by using difference observations
}

\author{
Christopher K. I. Williams \\ School of Informatics, University of Edinburgh, Edinburgh EH1 2QL, UK \\ c.k.i.williams@ed.ac.uk http://anc.ed.ac.uk \\ June 7, 2004
}

\begin{abstract}
In many areas of data modelling it is the case that observations at different locations (e.g. time frames or pixel locations) are augmented by differences of nearby observations (e.g. $\delta$-features in speech recognition, Gabor jets in image analysis). These augmented observations are then often modelled as being independent-how can this make sense? We provide two interpretations, showing (1) that the likelihood of data generated from an autoregressive (AR) process can be computed in terms of "independent" augmented observations, and (2) that the augmented observations can be given a coherent treatment in terms of the Products of Experts model (Hinton, 1999).
\end{abstract}

In automatic speech recognition it is often the case that Hidden Markov mod- 
els (HMMs) are used on observation vectors that are augmented by difference observations (so-called $\delta$ features), see Furui (1986). Under the HMM each observation vector is modelled as being conditionally independent given the hidden state. How can this make sense, as close-by differences are clearly not independent? A similar difficulty arises in image analysis tasks such as texture segmentation, see e.g. Dunn and Higgins (1995). Here derivative features obtained e.g. from Gabor filters or wavelet analysis are modelled as being independent at different locations, despite the fact that these features will have been computed sharing some pixels in common.

In this paper we present two solutions to this problem. In section 1 we show that if the data is generated from a vector autoregressive (AR) model then the likelihood can be expressed in terms of "independent" difference observations. In section 2 we show that the local models at each location can be combined using a Product of Experts model (Hinton, 1999) to provide a well-defined joint model for the data, and that this can be related to AR models. Section 3 discusses how these interpretations are affected if the local models are conditional on a hidden state variable, as is the case e.g. for HMMs.

\section{An AR model}

Consider a temporal vector autoregressive model

$$
\mathbf{X}_{t}=\sum_{i=1}^{p} A_{i} \mathbf{X}_{t-i}+\mathbf{N}_{t}
$$


where the $A_{i}$ 's are square matrices and $\mathbf{N}_{t}$ is iid Gaussian noise $\sim N\left(\mathbf{0}, \Sigma_{\mathbf{N}}\right) . \mathbf{X}_{t}$ and $\mathbf{N}_{t}$ have dimension $D$ for all $t$. To avoid complicated end effects we will use periodic (wrap-around) boundary conditions, so that the subscript $t-i$ should be read $\bmod (t-i, N)$. Thus there are $N$ random variables $\mathbf{X}_{0}, \ldots, \mathbf{X}_{N-1}$ which collectively we denote as $\mathbf{X}$, and similarly for $\mathbf{N}$. Then $\mathbf{X}$ and $\mathbf{N}$ are related by $\mathbf{N}=T \mathbf{X}$ for an appropriate matrix $T$. Thus

$$
\begin{aligned}
P(\mathbf{X}) & \propto \prod_{t=0}^{N-1} \exp \left\{-\frac{1}{2} \mathbf{N}_{t}^{T} \Sigma_{\mathbf{N}}^{-1} \mathbf{N}_{t}\right\} \\
& =\prod_{t=0}^{N-1} \exp \left\{-\frac{1}{2}\left[\sum_{i=0}^{p} A_{i} \mathbf{X}_{t-i}\right]^{T} \Sigma_{\mathbf{N}}^{-1}\left[\sum_{i=0}^{p} A_{i} \mathbf{X}_{t-i}\right]\right\}
\end{aligned}
$$

where we have set $A_{0}=-I$ so that $\mathbf{N}_{t}=-\sum_{i=0}^{p} A_{i} \mathbf{X}_{t-i}$.

Now let $\mathbf{Y}_{t}^{0}, \ldots, \mathbf{Y}_{t}^{p}$ be linearly independent linear combinations of $\mathbf{X}_{t}, \ldots \mathbf{X}_{t-p}$. For example we could choose $\mathbf{Y}_{t}^{0}=\mathbf{X}_{t}, \mathbf{Y}_{t}^{1}=\mathbf{X}_{t}-\mathbf{X}_{t-1}$ etc. As the $\mathbf{Y}_{t}^{i}$ 's are simple linear combinations of $\mathbf{X}_{t}, \ldots \mathbf{X}_{t-p}$ we have

$$
\sum_{i=0}^{p} A_{i} \mathbf{X}_{t-i}=\sum_{i=0}^{p} B_{i} \mathbf{Y}_{t}^{i}
$$

for some set of matrices $B_{i}$. So we can now write

$$
P(\mathbf{X}) \propto \prod_{t=0}^{N-1} \exp \left\{-\frac{1}{2}\left[\sum_{i=0}^{p} B_{i} \mathbf{Y}_{t}^{i}\right]^{T} \Sigma_{\mathbf{N}}^{-1}\left[\sum_{i=0}^{p} B_{i} \mathbf{Y}_{t}^{i}\right]\right\}
$$

showing that the likelihood of the underlying $\mathbf{X}$ process can be expressed in terms of a product of terms involving the difference observations up to order $p$ 
at each time. Stacking $\mathbf{Y}_{t}^{0}, \mathbf{Y}_{t}^{1}, \ldots, \mathbf{Y}_{t}^{p}$ as the vector $\mathbf{Y}_{t}$ we have

$$
P(\mathbf{X}) \propto \prod_{t=0}^{N-1} \exp \left\{-\frac{1}{2} \mathbf{Y}_{t}^{T} M \mathbf{Y}_{t}\right\}
$$

where the $(i, j)$ block of the matrix $M$ (between $\mathbf{Y}_{t}^{i}$ and $\mathbf{Y}_{t}^{j}$ ) has the form $B_{i}^{T} \Sigma_{\mathbf{N}}^{-1} B_{j}$. Equation 6 almost looks like a product of independent Gaussians, but note that $M$ is singular (it has rank $D$ as it arises from $\mathbf{N}_{t}$ ) so the correct normalization factor of the Gaussian cannot be obtained from it.

As a simple example, consider the scalar $\mathrm{AR}(1)$ process $X_{t}=\alpha X_{t-1}+N_{t}$ and set $Y_{t}^{0}=X_{t}, Y_{t}^{1}=X_{t}-X_{t-1}$. Thus

$$
\begin{aligned}
X_{t}-\alpha X_{t-1} & =(1-\alpha) X_{t}+\alpha\left(X_{t}-X_{t-1}\right) \\
& =(1-\alpha) Y_{t}^{0}+\alpha Y_{t}^{1} .
\end{aligned}
$$

To obtain the likelihood for the sequence $X$ the matrix $M$ will have the form

$$
M=\frac{1}{\sigma_{n}^{2}}\left(\begin{array}{cc}
(1-\alpha)^{2} & \alpha(1-\alpha) \\
\alpha(1-\alpha) & \alpha^{2}
\end{array}\right)
$$

where $\sigma_{n}^{2}=\operatorname{var}\left(N_{t}\right)$. As expected $M$ has rank 1 (it is an outer product).

Interestingly, the matrix $M$ is not equal to the inverse covariance of the $\mathbf{Y}_{t}$ 's derived from the distribution for $\mathbf{X}$. To show this we first use the result that for the scalar $\mathrm{AR}(1)$ process on the circle the covariance $C[j]=\left\langle X_{t} X_{t-j}\right\rangle$ is given by

$$
C[j]=\frac{\sigma_{n}^{2}\left(\alpha^{|j|}+\alpha^{|N-j|}\right)}{\left(1-\alpha^{2}\right)\left(1-\alpha^{N}\right)} .
$$


Thus

$$
\operatorname{cov}\left(\mathbf{Y}_{t}\right)=\left(\begin{array}{cc}
\left\langle Y_{t}^{0} Y_{t}^{0}\right\rangle & \left\langle Y_{t}^{0} Y_{t}^{1}\right\rangle \\
\left\langle Y_{t}^{0} Y_{t}^{1}\right\rangle & \left\langle Y_{t}^{1} Y_{t}^{1}\right\rangle
\end{array}\right)=\left(\begin{array}{cc}
C[0] & (C[0]-C[1]) \\
(C[0]-C[1]) & 2(C[0]-C[1])
\end{array}\right)
$$

Inversion of $\operatorname{cov}\left(\mathbf{Y}_{t}\right)$ shows that it is not equal to $M$ as given in equation 9 . Notice that the joint distribution of $\mathbf{Y}_{0}, \ldots, \mathbf{Y}_{N-1}$ is singular.

If we take an $\mathrm{AR}$ process on the $\mathbf{X}$ variables then of course one can choose linear combinations of the $\mathbf{X}_{t} \mathrm{~s}$ that are truly independent by carrying out an eigenanalysis. (For the periodic boundary conditions described above and time invariant coefficients the eigenbasis would be the Fourier basis.) However, if we allow ourselves an overcomplete basis set then we have shown that the likelihood of $\mathbf{X}$ under the AR process can readily be computed using "independent" densities at each location.

Although we have given the derivation above using Gaussian noise, in fact the conclusion concerning expressing the likelihood of the $\mathbf{X}$ sequence in terms of a product of terms involving $\mathbf{Y}_{t}$ 's is independent of the form of the noise driving the AR process.

It is also possible to extend the AR model described above beyond the temporal one-dimensional chain. For example Abend et al. (1965) describe Markov mesh models in two-dimensions. A simple example of such a model is a "thirdorder" Markov mesh where $\mathbf{X}_{i, j}$ depends autoregressively on $\mathbf{X}_{i, j-1}, \mathbf{X}_{i-1, j-1}$ and $\mathbf{X}_{i-1, j}$. The same construction in terms of $\mathbf{Y}$ variables can be used in this case. 


\section{Product of Experts Interpretation}

At an individual location we have a model $P_{t}\left(\mathbf{Y}_{t}\right)$ for the augmented vector $\mathbf{Y}_{t}$. To define a joint distribution on $\mathbf{X}$ we set

$$
P(\mathbf{X})=\frac{1}{Z} \prod_{t} P_{t}\left(\mathbf{Y}_{t}\right)
$$

where $Z$ is a normalization constant (known in statistical physics as the partition function). This is the Product of Experts construction (Hinton, 1999). One can also think of this as a Markov Random Field construction where $P(\mathbf{X}) \propto$ $\exp -E(\mathbf{X})$ and $E(\mathbf{X})=-\sum_{t} \log P_{t}\left(\mathbf{Y}_{t}\right)$. If each $P_{t}\left(\mathbf{Y}_{t}\right)$ is Gaussian then $P(\mathbf{X})$ will also be Gaussian, and $Z=(2 \pi)^{N / 2}|C|^{1 / 2}$ where $C$ is the covariance matrix of $\mathbf{X}$.

Again we consider a simple example relating to a scalar AR(1) process, so $\mathbf{Y}_{t}=\left(X_{t}, X_{t}-X_{t-1}\right)^{T}$. Let

$$
P_{t}\left(\mathbf{Y}_{t}\right) \propto \exp -\frac{1}{2}\left\{a_{0} X_{t}^{2}+a_{1}\left(X_{t}-X_{t-1}\right)^{2}\right\}
$$

with $a_{0}, a_{1}>0$. Then we obtain the joint distribution

$$
P(\mathbf{X}) \propto-\frac{1}{2}\left\{a_{0} \sum_{t} X_{t}^{2}+a_{1} \sum_{t}\left(X_{t}-X_{t-1}\right)^{2}\right\}
$$

$C^{-1}$, the inverse covariance matrix of $\mathbf{X}$ is circulant with entries $a_{0}+2 a_{1}$ on the diagonal and $-a_{1}$ in the bands above and below the diagonal and in the NE and SW corners. For the $\mathrm{AR}(1)$ process $X_{t}=\alpha X_{t-1}+N_{t}$ with $N_{t} \sim N\left(0, \beta^{-1}\right)$ 
we obtain corresponding entries of $\beta\left(1+\alpha^{2}\right)$ on the diagonal and $-\beta \alpha$ off the diagonal. The overall scale of $a_{0}$ and $a_{1}$ has the same effect as $\beta$ in setting the variance of the process but $r \stackrel{\text { def }}{=} \frac{a_{0}}{a_{1}}=\frac{(1-\alpha)^{2}}{\alpha}$, so for any given $\alpha$ value there is a corresponding value of $r^{1}$.

For the Gaussian case with expert $t$ involving interactions between $\mathbf{X}_{t}$ and $\mathbf{X}_{t-p}$ we obtain a quadratic form with the same pattern of banding as in the inverse covariance matrix of an $\operatorname{AR}(p)$ process, but as above for some choices of parameters there may not be a corresponding AR process.

Again this construction can be extended to two (or more) dimensions. For example in $2 \mathrm{~d}$ we might consider the variable $\mathbf{X}_{i, j}$ and the differences to its four neighbours to the N, S, E, W to obtain a five-dimensional $\mathbf{Y}$ vector. Equation 12 with each expert being Gaussian then defines a Gaussian Markov Random Field over the lattice of $\mathbf{X}$ variables.

\section{Incorporating Hidden State}

In speech recognition using HMMs the $\mathbf{Y}_{t}$ s are modelled as conditionally independent given the discrete hidden variable $s_{t}$. We now consider how this affects the interpretations given above.

For interpretation 1 we now consider a switching $\operatorname{AR}(p)$ process or ARHMM (see e.g. Woodland, 1992), so that $\mathbf{X}_{t}$ depends on $\mathbf{X}_{t-1}, \ldots, \mathbf{X}_{t-p}$ and also $s_{t}$. For example, using Gaussian noise and setting $s_{t}=k$, we have $\mathbf{X}_{t} \sim$

\footnotetext{
${ }^{1}$ Interestingly for $r \in(-4,0)$ there are no corresponding values of $\alpha$. Note that $\alpha=0 \Rightarrow a_{1}=0$.
} 
$N\left(\sum_{i=1}^{p} A_{i}^{k} \mathbf{X}_{t-i}, \Sigma^{k}\right)$; notice that the AR model parameters now depend on the switching variable. However we can still write the prediction $\sum_{i=1}^{p} A_{i}^{k} \mathbf{X}_{t-i}$ as a linear combination of the $\mathbf{Y}_{t}^{i} \mathrm{~s}$ so the likelihood can be written in the form of "independent" contributions from the $\mathbf{Y}_{t} \mathrm{~s}$. Note that the usual forward and backward HMM recursions can be carried out for the AR-HMM.

For interpretation 2 we have the individual component densities $P_{t}\left(\mathbf{Y}_{t} \mid s_{t}\right)$, and the joint distribution

$$
P(\mathbf{X} \mid \mathbf{s})=\frac{1}{Z(\mathbf{s})} \prod_{t} P_{t}\left(\mathbf{Y}_{t} \mid s_{t}\right)
$$

where $\mathbf{s}=\left(s_{0}, \ldots, s_{N-1}\right)$. Notice that the normalization constant in general depends on $\mathbf{s}$ and thus when given $\mathbf{X}$ the computation of $P(\mathbf{X} \mid \mathbf{s})$ does not only depend on the component densities but also on $Z(\mathbf{s})$. However, if $P_{t}\left(\mathbf{Y}_{t} \mid s_{t}\right)$ is Gaussian and has the same covariance structure but different means depending on $s_{t}$ for all $t$ then $Z$ would turn out to be independent of $\mathbf{s}$.

While writing this paper I became aware of the work of Tokuda et al. (2003) who correctly derive the product of Gaussian experts construction conditional on s and note the general dependence of $Z(\mathbf{s})$ on s. They also observe that use of the Viterbi algorithm to find the state sequence $\mathbf{s}$ that maximizes $P(\mathbf{s}) \prod_{t} P_{t}\left(\mathbf{Y}_{t} \mid s_{t}\right)$ (which is easily done with standard dynamic programming techniques) will not, in general, yield the sequence that maximizes $P(\mathbf{s} \mid \mathbf{X})$, because of the $Z(\mathbf{s})$ term.

Most practical HMM-based speech recognition systems use mixtures of Gaussians to model the $\mathbf{Y}_{t} \mathrm{~s}$ at each frame. The product of experts interpretation readily handles this situation. For an AR model interpretation, the use of a 
mixture distribution for the $\mathbf{Y}_{t} \mathrm{~s}$ already suggests a switching AR-process with the switching variable hidden.

\section{Discussion}

Above we have described both conditionally specified models (AR processes) and simultaneously specified models (products of experts) to define the joint density $P(\mathbf{X})^{2}$, and relate it to the augmented feature vectors $\left\{\mathbf{Y}_{t}\right\}$.

While this paper describes a theoretical framework for understanding why using difference observations make sense, it would be interesting to examine empirically the question of how well $\mathrm{AR}$ and PoE models do characterize the dependencies between time frames or pixel locations.

\section{Acknowledgements}

This note was inspired by questions raised by Joe Frankel's PhD thesis. Thanks to John Bridle and Joe Frankel for helpful conversations and comments on earlier drafts, to Joe Frankel for drawing my attention to Tokuda et al. (2003) and to the anonymous referees for their comments which helped to improve the paper.

\section{References}

Abend, K., Harley, T. J., and Kanal, L. N. (1965). Classification of Binary Random Patterns. IEEE Transactions on Information Theory, 11(4):538-544.

\footnotetext{
${ }^{2}$ This terminology is derived from Cressie (1993, section 6.3).
} 
Cressie, N. A. C. (1993). Statistics for Spatial Data. Wiley, New York.

Dunn, D. and Higgins, W. E. (1995). Optimal Gabor Filters for Texture Segmentation. IEEE Transactions on Image Processing, 4(7):947-964.

Furui, S. (1986). Speaker-independent isolated word recognition using dynamic features of speech spectrum. IEEE Transactions on Acoustics, Speech and Signal Processing, 34:52-59.

Hinton, G. E. (1999). Products of Experts. In Proceedings of the Ninth International Conference on Artificial Neural Networks, volume 1, pages 1-6.

Tokuda, K., Zen, H., and Kitamura, T. (2003). Trajectory Modelling based on HMMs with the Explicit Relationship between Static and Dynamic Features. In Proc. Eurospeech 2003.

Woodland, P. C. (1992). Hidden Markov Models using Vector Linear Prediction and Discriminative Output Distributions. In Proceedings of 1992 IEEE International Conference on Acoustics, Speech, and Signal Processing, volume I, pages 509-512. IEEE. 